\title{
Solvent Effect on Photoinitiator Reactivity in the Polymerization of 2-Hydroxyethyl Methacrylate
}

\author{
Iqbal Ahmad, ${ }^{1}$ Kefi Iqbal, ${ }^{2}$ Muhammad Ali Sheraz, ${ }^{1}$ Sofia Ahmed, ${ }^{1}$ Syed Abid Ali, ${ }^{3}$ \\ Sadia Hafeez Kazi, ${ }^{1}$ Tania Mirza, ${ }^{1}$ Raheela Bano, ${ }^{1}$ and Mohammad Aminuddin ${ }^{1}$ \\ ${ }^{1}$ Baqai Institute of Pharmaceutical Sciences, Baqai Medical University, 51 Deh Tor, Toll Plaza, \\ Super Highway, Gadap Road, Karachi 74600, Pakistan \\ ${ }^{2}$ Department of Dental Material Sciences, Baqai Dental College, Baqai Medical University, \\ 51 Deh Tor, Toll Plaza, Super Highway, Gadap Road, Karachi 74600, Pakistan \\ ${ }^{3}$ H.E.J. Research Institute of Chemistry, University of Karachi, Karachi 75270, Pakistan
}

Correspondence should be addressed to Muhammad Ali Sheraz; ali_sheraz80@hotmail.com

Received 26 September 2013; Revised 22 November 2013; Accepted 23 November 2013

Academic Editor: Francesco Paolucci

Copyright ( $\odot 2013$ Iqbal Ahmad et al. This is an open access article distributed under the Creative Commons Attribution License, which permits unrestricted use, distribution, and reproduction in any medium, provided the original work is properly cited.

Efficacy of photoinitiators such as riboflavin (RF), camphorquinone (CQ), and safranin $\mathrm{T}(\mathrm{ST})$ and triethanolamine as a coinitiator has been compared in carrying out the polymerization of 2-hydroxyethyl methacrylate (HEMA) in aqueous and organic solvents. HEMA solutions were polymerized in the presence of RF, CQ, and ST using a low intensity visible radiation source. HEMA was assayed by a UV spectrophotometric method during the initial stages of the reactions (i.e., $\sim 5 \%$ change). A comparison of the efficacy of photoinitiators in causing HEMA polymerization showed that RF is more efficient than CQ and ST. The rate of polymerization is directly related to solvent dielectric constant and inversely related to the solvent viscosity. RF is the most efficient photoinitiator in the polymerization of HEMA and the highest rate of reaction occurs in aqueous solutions. A general scheme for the polymerization of HEMA in the presence of photoinitiators is presented.

\section{Introduction}

The influence of solvent on the rates and mechanisms of chemical reactions is of great importance and has been discussed by many workers [1-5]. 2-Hydroxyethyl methacrylate (HEMA) is a component of resin-modified glass-ionomer cements used as restorative materials in dentistry. It undergoes polymerization in the presence of a photoinitiator during the setting process on bonding to the teeth [6]. The efficacy of photoinitiators in the polymerization of HEMA may be affected by medium characteristics including the polarity, viscosity, and the extent of radical formation involved in the reaction. Several studies have been carried out on the effect of solvent on the polymerization of HEMA using dilatometry $[7,8]$, gas chromatography [9], Raman spectroscopy [10], ATR-FTIR spectroscopy [11], and differential scanning calorimetry (DSC) [12]. The primary photochemical processes in polymerization may be dependent on the solvent and, therefore, the dielectric constant of the medium could affect the initial quantum yield of the process [8]. Most of the work on the polymerization of HEMA in aqueous solution has been carried out using water-soluble photoinitiators and information is lacking on their behavior in organic solvents. It would be worthwhile to evaluate the efficiency of these photoinitiators in the polymerization of HEMA in organic solvents. The present work is based on a study of the effect of solvent dielectric constant and viscosity on the rate of polymerization of HEMA in aqueous and organic solvents using a UV spectrophotometric method. Riboflavin $\left(\lambda_{\max }=445 \mathrm{~nm}\right)$ [13], camphorquinone $\left(\lambda_{\max }=\right.$ $468 \mathrm{~nm})$ [14], and safranin $\mathrm{T}\left(\lambda_{\max }=532 \mathrm{~nm}\right)$ [15] have been used as photoinitiators and triethanolamine as a coinitiator $[7,8,16]$ in the reaction. This work throws light on the effect of solvent characteristics, interactions, and kinetics of HEMA polymerization. A comparative study of the reactivity of different photoinitiators in aqueous and organic solvents 
<smiles>C=C(C)C(=O)OCCO</smiles>

2-Hydroxyethyl methacrylate<smiles>CC12CCC(C(=O)C1=O)C2(C)C</smiles>

Camphorquinone<smiles>[R10][R16]([H])([H])[C@H](CO)[C@@H](O)[C@@H](O)Cn1c2nc(=O)[nH]c(=O)c-2nc2cc(C)c(C)cc21</smiles><smiles></smiles><smiles>OCCN(CCO)CCO</smiles>

FIGURE 1: Chemical structures of HEMA and photoinitiators.

highlights the effect of solvent on the kinetics and mode of polymerization reactions. The chemical structures of HEMA and the photoinitiators used in this study are shown in Figure 1.

\section{Experimental}

2.1. Materials. Riboflavin (RF), camphorquinone (CQ), and safranin T (ST) were obtained from Sigma. Triethanolamine (TEOHA, Sigma) and 2-hydroxyethyl methacrylate (HEMA, Aldrich) were distilled under reduced pressure before use. Water was purified using a Millipore Milli-Q system.

2.2. Method of Polymerization. Polymerization of HEMA (monomer/solvent ratio 1.21:10,1 M) was carried out in the presence of photoinitiators, RF, CQ, and ST (absorbance of each photoinitiator at the $\lambda_{\max }$ was not more than 0.125 to avoid inhomogenous free radical distribution) [17], and $0.01 \mathrm{M}$ TEOHA as a coinitiator in aqueous and organic solvents under anaerobic conditions at $25^{\circ} \mathrm{C}$. The solutions were irradiated with a low intensity General Electric $15 \mathrm{~W}$ fluorescent lamp (emission in the visible region) fixed horizontally at a distance of $25 \mathrm{~cm}$ from the center of the vessel.

2.3. Spectral Measurements. All spectral measurements on fresh and polymerized solutions of HEMA were carried out on a Shimadzu UV-1601 recording spectrophotometer using quartz cells of $10 \mathrm{~mm}$ path length.

2.4. Fluorescence Measurements. Fluorescence measurements of RF in various HEMA solutions were carried out at room temperature $\left(\sim 25^{\circ} \mathrm{C}\right)$ using a SpectraMax 5 fluorimeter (Molecular Devices, USA) in the end point mode using $\lambda_{\text {ex }}=374 \mathrm{~nm}$ and $\lambda_{\text {em }}=520 \mathrm{~nm}$ [18]. The fluorescence was recorded in relative fluorescence units using a pure $0.05 \mathrm{mM}$ RF solution as standard.

2.5. Measurement of the Light Intensity. The measurement of the intensity of General Electric $15 \mathrm{~W}$ fluorescent lamp was carried out by potassium ferrioxalate actinometry [19] and a value of $2.85 \pm 0.26 \times 10^{16}$ quanta s$^{-1}$ was obtained. 
TABLE 1: Apparent first-order rate constants $\left(k_{\mathrm{obs}}\right)$ for the polymerization of HEMA in water and organic solvents.

\begin{tabular}{lcccc}
\hline \multirow{2}{*}{ Solvent } & \multirow{2}{*}{ Dielectric constant $^{\mathrm{a}}\left(25^{\circ} \mathrm{C}\right)$} & \multicolumn{2}{c}{$k_{\mathrm{obs}} \times 10^{4}\left(\mathrm{~s}^{-1}\right)^{\mathrm{b}}$} & \multicolumn{2}{c}{$\mathrm{CQ}$} & \multicolumn{2}{c}{$\mathrm{ST}$} \\
\hline Water & 78.5 & 1.000 & 5.05 & 4.04 \\
Acetonitrile & 37.5 & 2.898 & 3.71 & 3.02 \\
Methanol & 32.6 & 1.838 & 3.53 & 2.98 \\
Ethanol & 24.3 & 0.931 & 3.12 & 2.10 \\
1-Propanol & 20.1 & 0.514 & - & 1.93 \\
1-Butanol & 17.1 & 0.393 & - & 2.62 \\
\hline
\end{tabular}

${ }^{\mathrm{a}}$ CRC Handbook of Chemistry and Physics, 90th edition, CRC Press, Boca Raton, FL, 2010.

${ }^{\mathrm{b}}$ The values of rate constants are relative and depend on specific experimental conditions including light intensity.

2.6. Assay of HEMA. The assay of HEMA in fresh and polymerized solutions was carried out by mixing a small amount of the solution with $0.05 \mathrm{M}$ phosphate buffer, $\mathrm{pH}$ 7.0, and measurement of absorbance at $208 \mathrm{~nm}$ (molar absorptivity $8000 \mathrm{M}^{-1} \mathrm{~cm}^{-1}$ ). At this dilution the photoinitiator has negligible absorption at the analytical wavelength. The concentration of the samples was calculated using the following least squares regression equation: $y=0.9920 x+0.0012 ; r^{2}=$ 0.9996 . The validity of Beer's law has been confirmed in the concentration range of $0.1-1.0 \times 10^{-4} \mathrm{M}$ HEMA. The RSD of the assay method is within $\pm 3 \%$. The method has previously been used for the study of the photoinitiated polymerization of HEMA by RF/TEOHA system in aqueous solution [20].

\section{Results and Discussion}

3.1. Effect of Dielectric Constant on Polymerization. The rate of the reactions between dipolar molecules is dependent on the dielectric constant, $D$, of the medium [4]. Consider

$$
\ln k=\ln k_{D=\infty}-K\left(\frac{1}{D}\right)
$$

where $k_{D=\infty}$ is the rate constant in a medium of infinite dielectric constant and $K$ is a constant involving terms such as ion charge and distance between ions. The dielectric constant of the medium is approximately equal to the dielectric constant of the solvent in dilute solutions. A decrease in dielectric constant of the medium tends to decrease the rate of reaction and conversely. The effect of dielectric constant on the polymerization of HEMA has been evaluated in the presence of different photoinitiators.

3.1.1. Riboflavin as Photoinitiator. The polymerization of HEMA was carried out in aqueous and organic solvents (acetonitrile, methanol, and ethanol) containing RF as photoinitiator and $0.01 \mathrm{M}$ TEOHA as a coinitiator. The reactions in 1-propanol and 1-butanol could not be carried out due to the insolubility of RF in these solvents. The analytical data were subjected to kinetic treatment and the reactions were found to follow pseudo first-order kinetics in the initial stages ( $5 \%$ HEMA loss) using a low intensity radiation source. The steady-state assumption of the rate of initiation being equal to the rate of termination in polymerization reactions is considered valid only at a low conversion of monomer [14]

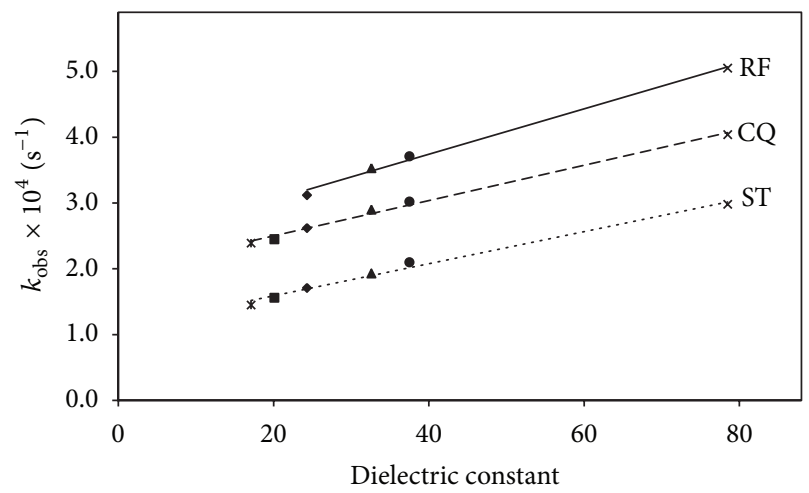

FIGURE 2: Plots of $k_{\mathrm{obs}}$ for polymerization of HEMA against dielectric constant. $\times$ : water; $\bullet$ : acetonitrile; $\mathbf{\Lambda}$ : methanol; $\$$ : ethanol; 匹: 1-propanol; *: 1-butanol.

and is represented by the apparent first-order rate constant $\left(k_{\text {obs }}\right)$ in this study. The values of $k_{\text {obs }}$ for the polymerization of HEMA in the presence of RF in water and organic solvents are reported in Table 1. In order to develop a correlation between $k_{\mathrm{obs}}$ and the dielectric constant of the medium, $D$, a plot of $k_{\text {obs }}$ versus dielectric constant of the solvents was constructed (Figure 2). It was observed that the rate of reaction is dependent upon the solvent and is a linear function of the dielectric constant of the medium. Since RF is used as a photoinitiator in this reaction, it is necessary to understand the behavior of RF on excitation. This could be explained on the basis of the existence of a polar flavin (Fl) intermediate, which would facilitate the polymerization reaction with an increase in the polarity of the medium. A strong evidence for the presence of such an intermediate has been presented by Ahmad and Tollin [21] who studied the solvent effect on flavin electron transfer reactions using laser flash photolysis. According to these workers the reduction of flavin triplet $\left({ }^{3} \mathrm{Fl}\right)$ by the substrate (amine in this case) proceeds via a dipolar intermediate in water and organic solvents and, therefore, the rate is increased with an increase in the solvent dielectric constant:

$$
{ }^{3} \mathrm{Fl}+\mathrm{AH} \longrightarrow\left(\mathrm{Fl}^{\delta-} \cdots \mathrm{H} \cdots \mathrm{A}^{\delta^{+}}\right)
$$

The extent of solvent interaction with the dipolar intermediate would determine the degree to which it leads to the 
TABLE 2: Fluorescence intensity of $1 \times 10^{-5} \mathrm{M} \mathrm{RF}$ in water and organic solvents.

\begin{tabular}{lc}
\hline Solvent & Relative fluorescence intensity at $520 \mathrm{~nm}$ \\
\hline Water & 100.0 \\
Ethanol & 87.1 \\
Methanol & 86.7 \\
Acetonitrile & 84.1 \\
\hline
\end{tabular}

formation of radicals. In these reactions the primary photochemical process is considered as being the electron transfer between reactants. In such a case the transition state is more polar than the reactant and the rate of reaction would increase with the dielectric constant of the medium as observed in the case of the photolysis of formylmethylflavin [22]. Thus the polarity of the medium in which the polymerization of HEMA is being carried out would exert an effect on the rate of the reaction, and the primary physical factor determining the observed dependence of $k_{\mathrm{obs}}$ on $D$ is the electrostatic interaction. It should, however, be noted that an alternative interpretation to the interrelation between $k_{\mathrm{obs}}$ and $D$ may be given; namely, the increase in solvent dielectric may lead to increase in the strength of solvophobic effect which is mainly of entropic nature and originates from change in the solvent structure around the reactants on complexation [23]. This may affect $k_{\text {obs }}$ on the state of HEMA polymerization and act together with the electrostatics. Further discussion of the contribution from these two factors to the dependence of $k_{\text {obs }}$ on $D$ falls out of the scope of the present paper. However, it is worth noting that $D$ is directly related to the strength of electrostatic interactions and only indirectly accounts for the solvophobic effect; hence, the linear interrelation between $k_{\text {obs }}$ and solvent dielectric in Figure 2, presumably, reflects the electrostatics as a major factor determining the observed dependence. Anyway, the obtained results suggest that water, with the highest dielectric constant, appears to be the best medium for carrying out polymerization of HEMA in the presence of RF to obtain a greater yield than that in the organic solvents.

It has been reported that the rates of polymerization of HEMA are decreased with a decrease in medium polarity, that is, from water to acetonitrile, as a result of singlet state quenching in organic solvents $[7,8]$. The results obtained in this study are in accordance with this behavior since the fluorescence of RF is reduced with the polarity of the medium (Table 2).

3.1.2. Camphorquinone as Photoinitiator. The results of the effect of solvent dielectric constant on the rate of polymerization of HEMA in the presence of CQ as photoinitiator may be considered on the basis of the data discussed above in the case of RF as a photoinitiator. The values of $k_{\mathrm{obs}}$ for these reactions are given in Table 1 . The polymerization behavior of HEMA in aqueous and organic solvents is similar to that observed in the presence of RF with respect to the effect of dielectric constant (Figure 2). However, the values of $k_{\text {obs }}$ in this case are lower than those observed for RF and may be due to a lower reactivity of the polar intermediate and subsequent radical formation in this reaction. In view of the structural consideration ( $\mathrm{C}=\mathrm{O}$ groups) the polar character of CQ would be lower than that of RF (a highly conjugated system), resulting in lower rate constants for the reactions. The effectiveness of CQ/TEOHA system depends on the $\mathrm{H}$-atom donor ability of the amine in a particular environment and subsequent interaction of the photoinitiator excited species with the monomer (HEMA) to undergo polymerization [11].

3.1.3. Safranin $T$ as Photoinitiator. The apparent firstorder rate constants for the polymerization of HEMA in ST/TEOHA system in aqueous and organic solvent are reported in Table 1. A plot of these rate constants as a function of solvents dielectric constant is shown in Figure 2. These results indicate that the reactivity of ST is lower than those of RF and CQ as photoinitiators. Apart from a consideration of the excited state polarization behavior of this molecule and polarity of the intermediate involved in this reaction, the visible absorption maximum $(532 \mathrm{~nm})$ of $\mathrm{ST}$ is higher than those of RF $(444 \mathrm{~nm})$ and CQ $(468 \mathrm{~nm})$. It would provide relatively less energy for the excitation of the molecule and would have a lower efficiency compared with the other two photoinitiators. Thus the rates of polymerization of HEMA in this case are lower than those of RF and CQ.

The slopes of the plots of $k_{\text {obs }}$ versus dielectric constant of the medium for the photoinitiators used are in the following order:

$$
\mathrm{RF}>\mathrm{CQ}>\mathrm{ST} \text {. }
$$

This indicates the magnitude of the solvent effect on the reactivity of these compounds in initiating the polymerization of HEMA.

3.2. Effect of Viscosity on Polymerization. Another important factor that may influence the rate of a chemical reaction is the viscosity of the medium. This appears to control the solute diffusion and hence the rate of a reaction. A pervious study has shown that the ${ }^{3} \mathrm{Fl}$ quenching by a substrate is proportional to the inverse of solvent viscosity as expected for a diffusional process [21]. The effect of viscosity on the polymerization of HEMA in the presence of different photoinitiators has been discussed in the following sections.

3.2.1. Riboflavin as Photoinitiator. Polymerization reactions of HEMA in water at 1,2 , and $3 \mathrm{M}$ concentrations in the presence of different photoinitiators have shown that the rates are decreased with an increase in the viscosity of the medium [20]. In order to confirm the effect of medium viscosity on the rate of these reactions, the values of $k_{\text {obs }}$ in organic solvents in the presence of RF were plotted as a function of the inverse of solvent viscosity (Table 1) and a linear relationship was observed as expected (Figure 3). These observations are also supported by the earlier data on the rate constants reported by Valdebenito and Encinas [8], where a decrease in fluorescence quantum yields of the photoinitiator in organic solvents compared to those in aqueous medium was observed. The decrease in fluorescence intensity of RF in organic solvents (Table 2) indicates the effect of solvent 


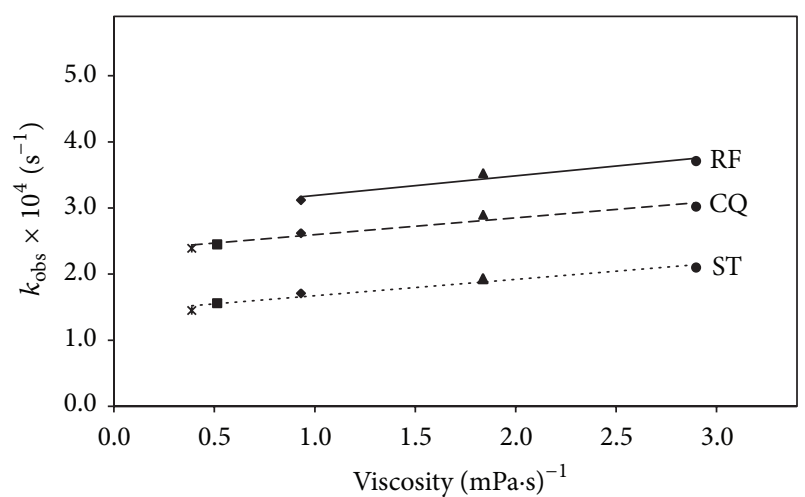

FIGURE 3: Plots of $k_{\text {obs }}$ for polymerization of HEMA against inverse of solvent viscosity. Symbols are the same as those in Figure 2.

viscosity (Figure 3) on the reaction. This may be explained on the basis of Fl singlet quenching in organic solvents as a result of change in viscosity. The radical-radical reactions, as in the case of polymerization of HEMA, are sensitive to solvent viscosity [5]. Moreover, the decrease in the rate of polymerization has also been ascribed to the combination of a monomer viscosity effect [24].

3.2.2. Camphorquinone as Photoinitiator. The effect of viscosity on the rate of polymerization of HEMA using CQ as a photoinitiator shows a similar behavior as observed in the case of RF. A plot of $k_{\mathrm{obs}}$ versus the inverse of solvent viscosity shows a linear relationship and the rates tend to decrease with an increase in the viscosity of the medium. This appears to be due to a decrease in solute diffusion processes with an increase in solvent viscosity. The slope of the plot (Figure 3) indicates that viscosity exerts a lower effect on the rates in the presence of CQ compared to that of RF.

3.2.3. Safranin $T$ as Photoinitiator. The results obtained with $\mathrm{ST}$ as a photoinitiator in the polymerization of HEMA are similar to those of RF and CQ. A plot of $k_{\mathrm{obs}}$ versus inverse of viscosity shows a linear relationship (Figure 3 ) and the rates are further lower than those observed in the case of CQ. The effect of viscosity on the rates of polymerization of HEMA using ST as a photoinitiator is lower than those of RF and CQ. Thus viscosity appears to play a significant role in the efficacy of polymerization processes.

3.3. Spectral and Structural Characteristics of Photoinitiators. In order to provide further explanation of the reactivity of the three photoinitiators (RF, CQ, and ST) used in this study, a consideration of the spectral and structural characteristics of these compounds is necessary.

RF exhibits an absorption maximum at $445 \mathrm{~nm}$ and undergoes $\pi-\pi^{*}$ transition resulting in high molar absorptivity $\left(12500 \mathrm{M}^{-1} \mathrm{~cm}^{-1}\right)$ [25]. It is a polar compound $\left(\mathrm{p} K_{a} 1.9\right.$, 10.2 ) and exists as a dipolar molecule in aqueous solution. It is known to produce a polar intermediate on light absorption [21] which would further lead to the formation of free radicals and then efficient interaction with the amine to

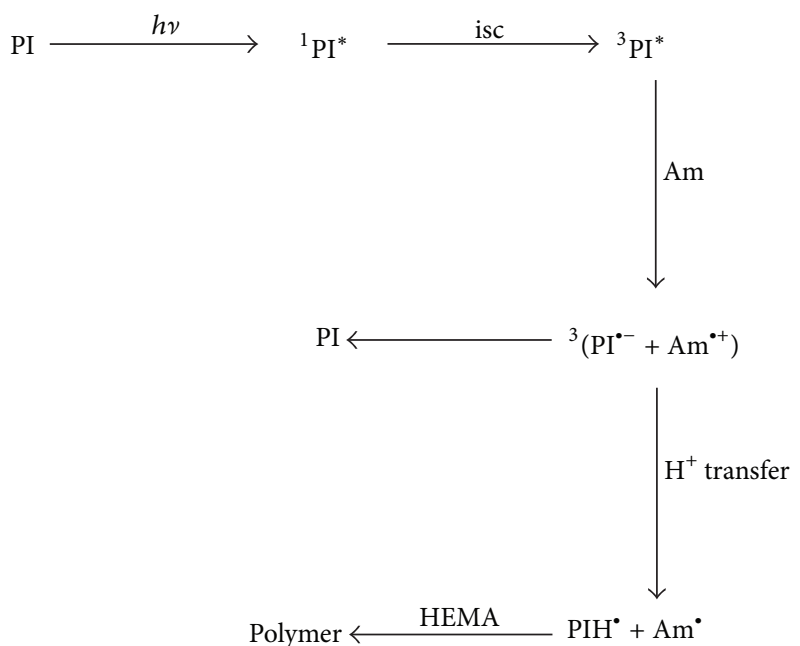

FIGURE 4: A general scheme for the polymerization of HEMA in the presence of photoinitiators.

initiate polymerization. Compared with RF, CQ possesses weakly ionizable $\mathrm{C}=\mathrm{O}$ groups. The light absorption at $468 \mathrm{~nm}$ would lead to $n-\pi^{*}$ transition of the dicarbonyl group in the molecule which has low molar absorptivity. Hence its efficiency in water would be lower compared to that of RF as observed. ST $\left(\mathrm{pK}_{a} 4.0\right)$ is an ionizable compound and exhibits an absorption maximum at $532 \mathrm{~nm}$ that results from $\pi-\pi^{*}$ transition. All these photoinitiators, on excitation, produce radicals which interact with the amine and thus initiate the polymerization of HEMA. The degree of interaction of these photoinitiators with TEOHA would depend on the yield of their radicals in aqueous and organic solvents. The rate of the reaction would depend on the viability of the radical pair in a specific medium leading to polymerization.

It needs to be emphasized that, under the assay conditions of HEMA, on the dilution of photolysed solutions, the maximum TEOHA concentration used $(0.01 \mathrm{M})$ would be too low to undergo complexation with HEMA in a molar ratio. The UV spectra of HEMA at such a dilution did not show any change in UV absorption in the presence of TEOHA. This suggests that there is no possibility of interaction between these compounds to affect the rate constants.

3.4. Mechanism of Polymerization. The mechanisms of polymerization of HEMA using RF [26], CQ [14], and ST [7] as photoinitiators have previously been reported and involve similar steps in radical formation and further interactions to yield the polymer. Based on these mechanisms a general scheme for the polymerization of HEMA in the presence of different photoinitiators is presented in Figure 4.

The photoinitiator (PI) on the absorption of light is promoted to the excited singlet state $\left({ }^{1} \mathrm{PI}^{*}\right)$ followed by intersystem crossing (isc) to the excited triplet state $\left({ }^{3} \mathrm{PI}^{*}\right)$. ${ }^{3} \mathrm{PI}^{*}$ is quenched by the amine (Am) by electron transfer to form a semireduced ${ }^{3} \mathrm{PI}^{\bullet-}$ and a semioxidized ${ }^{3} \mathrm{Am}^{\circ+}$ radical pair $\left[^{3}\left(\mathrm{PI}^{0^{-}}+\mathrm{Am}^{\bullet+}\right)\right]$. This is followed by proton transfer from the $\mathrm{Am}^{\circ+}$ radical to the $\mathrm{PI}^{\circ-}$ radical to produce neutral 
PI and Am radicals. The free radicals thus formed in the reaction would add to the double bonds of HEMA monomer and initiate the polymerization process. The rate and extent of polymerization would depend on the solvent polarity and viscosity.

\section{Conclusion}

The polymerization of HEMA in the presence of RF, CQ, and ST as photoinitiators and TEOHA as a coinitiator shows that RF is more efficient than CQ and ST. The rate of polymerization is a linear function of the solvent dielectric constant indicating the involvement of polar intermediates in the photoinitiated reaction. The effect of decrease in fluorescence intensity of RF on the rate of the reaction is due to a decrease in solvent polarity causing the quenching of the excited singlet state. The rate of the reaction is inversely proportional to the solvent viscosity as a result of the diffusion controlled process. The study highlights the role of solvent characteristics in the efficiency of the polymerization of HEMA in the presence of the photoinitiators used.

\section{Conflict of Interests}

There is no conflict of interests.

\section{References}

[1] E. S. Amis and J. F. Hinton, Solvent Effects on Chemical Phenomena, Academic Press, New York, NY, USA, 1973.

[2] C. Reichardt, Solvents and Solvent Effect in Organic Chemistry, Wiley-VCH, New York, NY, USA, 2nd edition, 1988.

[3] E. Buncel, R. A. Stairs, and H. Wilson, The Role of the Solvent in Chemical Reactions, Oxford University Press, New York, NY, USA, 2003.

[4] P. J. Sinko, Martin's Physical Pharmacy and Pharmaceutical Sciences, Lippincott Williams \& Wilkins, Philadelphia, Pa, USA, 5th edition, 2006.

[5] N. J. Turro, V. Ramamurthy, and J. S. Scaiano, Modern Molecular Photochemistry of Organic Molecules, University Science Books, Sausalito, Calif, USA, 1st edition, 2010.

[6] J. W. Nicholson, The Chemistry of Medical and Dental Materials, The Royal Society, Cambridge, UK, 2002.

[7] M. V. Encinas, A. M. Rufs, M. G. Neumann, and C. M. Previtali, "Photoinitiated vinyl polymerization by safranine T/triethanolamine in aqueous solution," Polymer, vol. 37 , no. 8 , pp. 1395-1398, 1996.

[8] A. Valdebenito and M. V. Encinas, "Photopolymerization of 2hydroxyethyl methacrylate: effect of the medium properties on the polymerization rate," Journal of Polymer Science A, vol. 41, no. 15, pp. 2368-2373, 2003.

[9] K. L. Beers, S. Boo, S. G. Gaynor, and K. Matyjaszewski, "Atom transfer radical polymerization of 2-hydroxyethyl methacrylate," Macromolecules, vol. 32, no. 18, pp. 5772-5776, 1999.

[10] Y. Wang, P. Spencer, X. Yao, and Q. J. Ye, "Effect of coinitiator and wafer on the photoreactivity and photopolymerization of HEMA/camphoquinone-based reactant mixtures," Journal of Biomedical Materials Research A, vol. 78, no. 4, pp. 721-728, 2006.
[11] X. Guo, Y. Wang, P. Spencer, Q. Ye, and X. Yao, "Effects of water content and initiator composition on photopolymerization of a model BisGMA/HEMA resin," Dental Materials, vol. 24, no. 6, pp. 824-831, 2008.

[12] E. Andrzejewska, M. Podgorska-Golubska, I. Stepniak, and M. Andrzejewski, "Photoinitiated polymerization in ionic liquids: kinetics and viscosity effects," Polymer, vol. 50, no. 9, pp. 20402047, 2009.

[13] P. F. Heelis, “The photophysical and photochemical properties of flavins (isoalloxazines)," Chemical Society Reviews, vol. 11, no. 1, pp. 15-39, 1982.

[14] J. Jakubiak, X. Allonas, J. P. Fouassier et al., "Camphorquinoneamines photoinitating systems for the initiation of free radical polymerization," Polymer, vol. 44, no. 18, pp. 5219-5226, 2003.

[15] C. M. Previtali, S. G. Bertolotti, M. G. Neumann, I. A. Pastre, A. M. Rufs, and M. V. Encinas, "Laser flash photolysis study of the photoinitiator system safranine T-aliphatic amines for vinyl polymerization," Macromolecules, vol. 27, no. 25, pp. 7454-7458, 1994.

[16] M. V. Encinas, A. M. Rufs, S. G. Bertolotti, and C. M. Previtali, "Xanthene dyes/amine as photoinitiators of radical polymerization: a comparative and photochemical study in aqueous medium," Polymer, vol. 50, no. 13, pp. 2762-2767, 2009.

[17] J. Alvarez, E. A. Lissi, and M. V. Encinas, "Effect of the initiator absorbance on the transition-metal complex photoinitiated polymerization," Journal of Polymer Science A, vol. 36, no. 1, pp. 207-208, 1998.

[18] P. S. Song and D. E. Metzler, "Photochemical degradation of flavins. IV. Studies of the anaerobic photolysis of riboflavin," Photochemistry and Photobiology, vol. 6, no. 10, pp. 691-709, 1967.

[19] C. G. Hatchard and C. A. Parker, "A new sensitive chemical actinometer. II. Potassium ferrioxalate as a standard chemical actinometer," Proceedings of Royal Society London A, vol. 235, no. 1203, pp. 518-536, 1956.

[20] I. Ahmad, K. Iqbal, M. A. Sheraz et al., "Photoinitiated polymerization of 2-hydroxyethyl methacrylate by Riboflavin/Triehanolamine in aqueous solution: a kinetic study,' ISRN Pharmaceutics, vol. 2013, Article ID 958712, 7 pages, 2013.

[21] I. Ahmad and G. Tollin, "Solvent effects of flavin electron transfer reactions," Biochemistry, vol. 20, no. 20, pp. 5925-5928, 1981.

[22] I. Ahmad, Q. Fasihullah, and F. H. M. Vaid, "Photolysis of formylmethylflavin in aqueous and organic solvents," Photochemical and Photobiological Sciences, vol. 5, no. 7, pp. 680-685, 2006.

[23] P. Maurel, "Relevance of dielectric constant and solvent hydrophobicity to the organic solvent effect in enzymology," The Journal of Biological Chemistry, vol. 253, no. 5, pp. 1677-1683, 1978.

[24] J. D. Biasutti, G. E. Roberts, F. P. Lucien, and J. P. A. Heuts, "Substituent effects in the catalytic chain transfer polymerization of 2-hydroxyethyl methacrylate," European Polymer Journal, vol. 39, no. 3, pp. 429-435, 2003.

[25] I. Ahmad, Q. Fasihullah, and F. H. M. Vaid, "A study of simultaneous photolysis and photoaddition reactions of riboflavin in aqueous solution," Journal of Photochemistry and Photobiology $B$, vol. 75, no. 1-2, pp. 13-20, 2004. 
[26] B. Orellana, A. M. Rufs, M. V. Encinas, C. M. Previtali, and S. Bertolotti, "The photoinitiation mechanism of vinyl polymerization by riboflavin/triethanolamine in aqueous medium," Macromolecules, vol. 32, no. 20, pp. 6570-6573, 1999. 

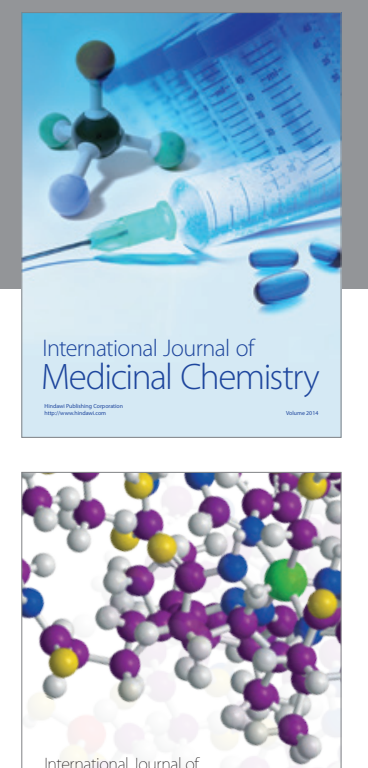

\section{Carbohydrate} Chemistry

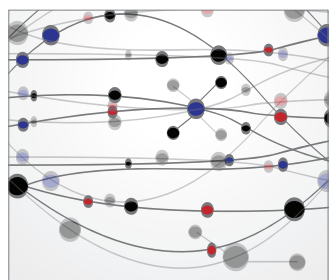

The Scientific World Journal
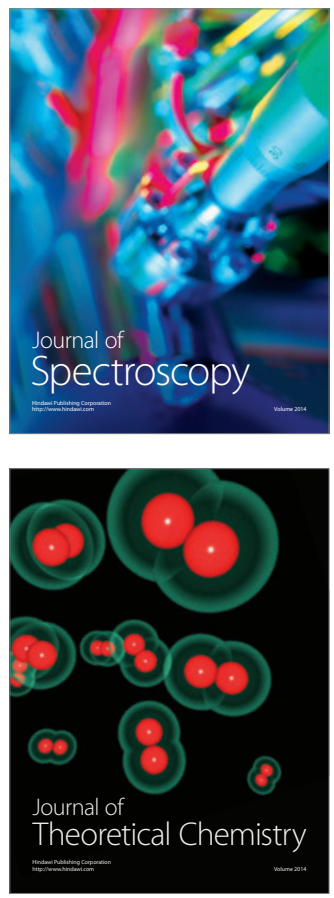
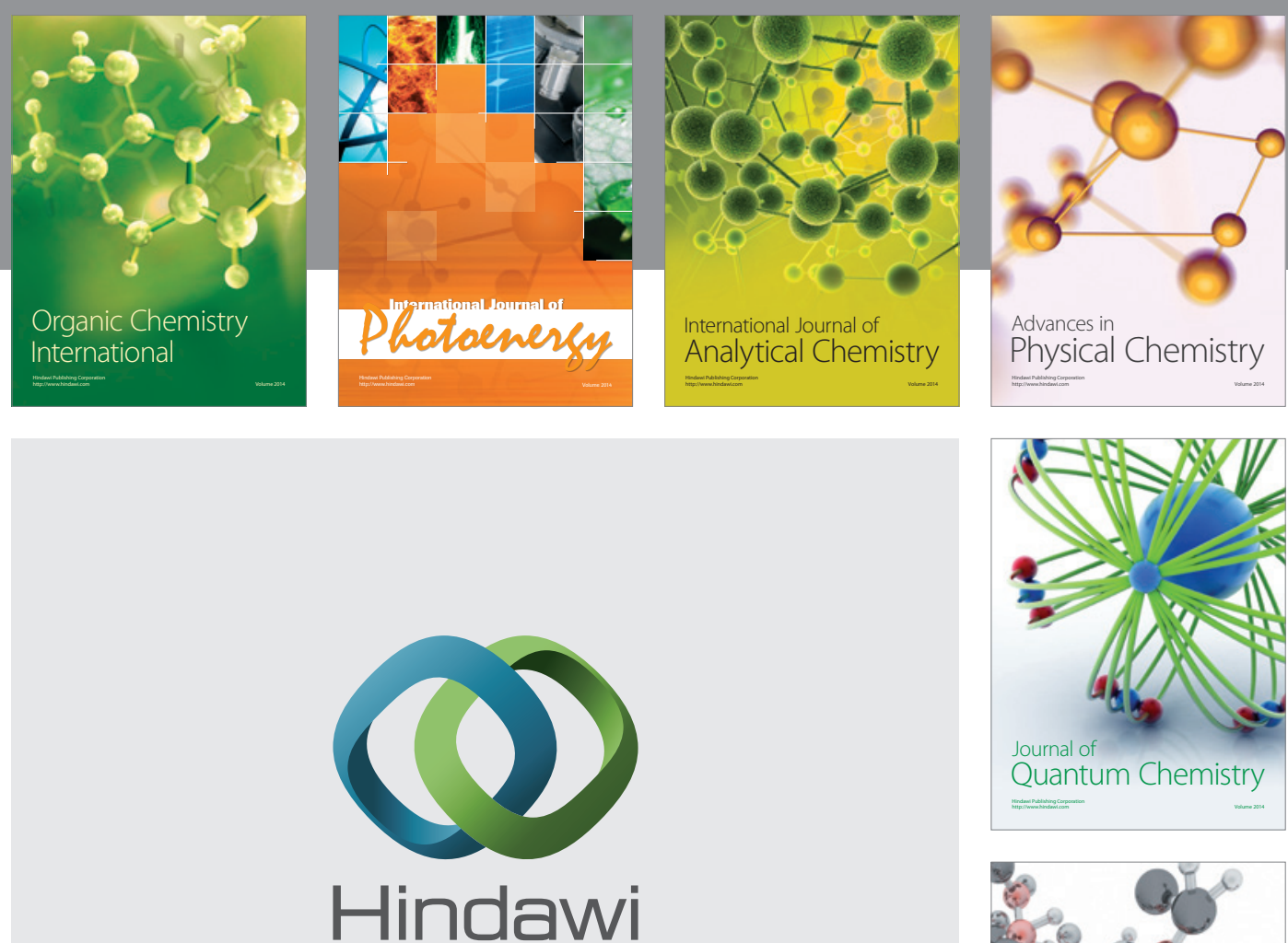

Submit your manuscripts at

http://www.hindawi.com

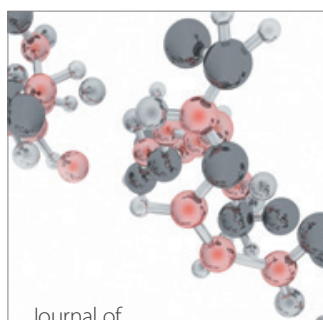

Analytical Methods

in Chemistry

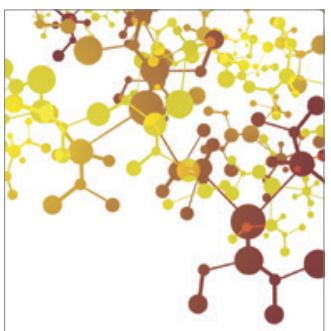

Journal of

Applied Chemistry

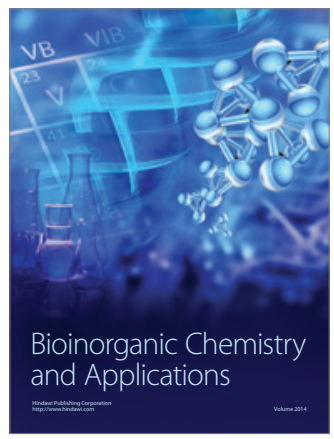

Inorganic Chemistry
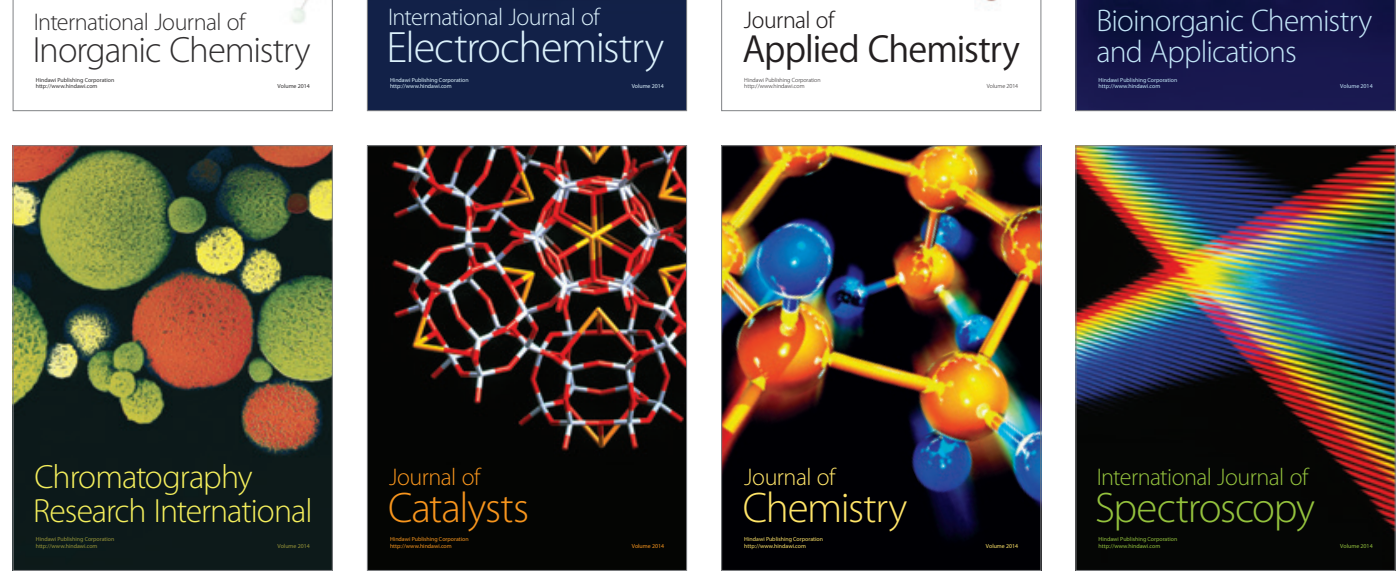